Revista Ingeniería Biomédica

ISSN 1909-9762 / Volumen 9 / Número 18 / Julio-diciembre de 2015 / pp. 139-144

Escuela de Ingeniería de Antioquia-Universidad CES / Envigado, Colombia

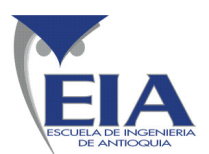

Ser. Saber y Serwir

\title{
Modelo de capacitación de tecnología biomédica para clínicas y hospitales de tercer nivel, enfocado en personal asistencial
}

\author{
G. A. Franco², D. Jaramillo², J. G. Barreneche $e^{1,2}$ \\ ${ }^{1}$ Grupo de Investigación en Bioinstrumentación e Ingeniería Clínica GIBIC. Medellín, Colombia \\ ${ }^{2}$ Department of Bioengineering, Universidad de Antioquia. Medellin, Colombia
}

Recibido 1 de julio de 2015. Aceptado 15 de agosto de 2015

\section{Training Model for Clinical and Biomedical Technology for Tertiary Hospitals, Focused on Caregivers}

\author{
MODELO DE TREINAMENTO DE TECNOLOGIA BIOMÉDICA PARA HOSPITAIS E CLÍNICAS TERCIÁRIOS, COM
}

FOCO EM PROFISSIONAIS DE SAÚDE

\begin{abstract}
Resumen - El proceso de capacitación en tecnología biomédica se enmarca dentro de los requerimientos del decreto \# 4725 de 2005 emitido por el ministerio de protección social, y es parte del proceso de adquisición de tecnología el cual es de gran importancia para las instituciones prestadoras de salud en Colombia. La tecnología médica requiere procesos detallados de capacitación. Uno de los aspectos más importantes a considerar más no el único es la clasificación de riesgo de los dispositivos médicos, el cual es un factor muy importante porque puede cambiar el tiempo y costo de la capacitación. Este artículo pretende hacer una revisión de algunos modelos de capacitación en clínicas y hospitales y comprarlos entre sí, Adicionalmente el artículo presenta un modelo que se basa en los criterios más representativos de los modelos analizados.

Palabras clave - Evaluación de tecnología; dispositivos médicos; tecnología biomédica; entrenamiento de tecnología; ciclo de vida; ingeniería clínica; riesgo de tecnología biomédica; gestión tecnológica.

Abstract - The training in biomedical technology is part of the requirements of the decree \# 4725 of 2005 issued by the Colombian Ministry of Social Protection, and it's part of the technology procurement process which is of great importance to the health institutions in Colombia. Medical technology requires detailed training processes. One of the most important aspects to consider, not the only one, is risk classification of medical devices, which is a very important factor that may affect time and cost of training. This article aims to review and compare some models of training in clinics and hospitals. In addition, the paper introduces a model based on the most representative criteria of the models analyzed.
\end{abstract}

Keywords - Technology Evaluation; Medical Devices; Biomedical Technology; Technology Training; Life Cycle; Clinical Engineering; Biomedical Technology Risk; Technological Management.

\% Dirección para correspondencia: juan.barreneche@udea.edu.co

DOI: http:/dx.doi.org/10.14508/rbme.2015.9.18.139-144 
Resumo -O treinamento em tecnologia biomédica é parte dos requisitos do decreto \# 4725 de 2005 emitido pelo Ministério da Proteção Social, e faz parte do processo de aquisição de tecnologia, que é de grande importância para as instituições de saúde na Colômbia. A tecnologia médica requer processos de treinamento detalhados. Um dos aspectos mais importantes a considerar não é a única classificação de risco de dispositivos médicos, o que é um fator muito importante que pode mudar o tempo e o custo do treinamento. Este artigo tem por objetivo revisar alguns modelos de formação em clínicas e hospitais e compará-los entre si, Além disso, o artigo apresenta um modelo com base nos critérios mais representativos dos modelos analisados.

Palavras chave - Avaliação tecnológica; Dispositivos médicos; Tecnologia biomédica; Tecnologia treinamento; Ciclo de vida; Engenharia clínica; Risco tecnologia biomédica; Gestão tecnológica.

\section{INTRODUCCIÓN}

$E^{n}$ las instituciones prestadoras de servicios en salud, es necesario una continua capacitación, a causa de las diferentes rotaciones de personal, la adquisición de nuevas tecnologías o la falta de conocimiento por parte del personal asistencial (médico y de enfermería) acerca del uso de los dispositivos médicos.

En Inglaterra el 6\% de 2010 pacientes entre el año 2010 y el 2012, fueron declarados muertos a causa de una falla del equipo, un mal uso o mala lectura de los profesionales que lo operan [1], siendo esto una evidencia de la necesidad de capacitar al personal continuamente sobre tecnología que tienen a su disposición.

Es por esto que la institución debe elaborar un protocolo de capacitación en donde el personal asistencial tenga información acerca de la nueva tecnología o de la tecnología ya existente. Además, se debe garantizar que este personal asista a la capacitación y adquiera el conocimiento necesario para evitar fallas en el diagnóstico y riesgo en la vida del paciente [2].

De esta manera se debe elaborar un plan estructurado para la capacitación continua en las clínicas y hospitales, para esto, se realizó un estudio de dos modelos de capacitación, uno nacional y otro internacional, con el fin de buscar buenas prácticas de aquellos. A partir de este estudio, se planteó un modelo de capacitación, en el que se busca presentar la mejor manera de realizar una capacitación dentro de una entidad prestadora de servicios en salud, teniendo en cuenta aspectos relevantes, como las herramientas usadas para la capacitación, el tiempo necesario para la realización de esta y el riesgo de la tecnología medica, que deben de tener las propias instituciones con el fin de brindar un óptimo servicio y una seguridad al paciente.

\section{Metodología}

Se ejecutó en tres fases, en la primera fase corresponde a una búsqueda bibliográfica de los temas relacionados al problema planteado, es decir, los conceptos básicos de capacitación al personal asistencial, enfatizados principalmente en la norma Colombiana del decreto 4725 de 2005, Además, se realizó una búsqueda de los modelos de capa- citación aplicados a clínicas y hospitales a nivel mundial con el objetivo de realizar una comparación entre estos.

En la segunda fase se eligen los parámetros que cumplan con las condiciones actuales del país, con base a las necesidades requeridas por la institución prestadora de servicios en salud, teniendo en cuenta las ventajas y desventajas de los modelos ya existentes.

Finalmente, en la tercera fase, de acuerdo a los parámetros obtenidos en la segunda fase, se plantea un modelo que permita mejorar los modelos ya existentes de capacitación de tecnología médica utilizados en clínicas y hospitales de Colombia. Para esto se debe tener en cuenta si es una tecnología nueva, gestionar la capacitación por parte del proveedor, y si es una tecnología ya existente y el riesgo de la tecnología.

Este modelo debe tener en cuenta ciertos factores tales como [3]:

- La detección de las necesidades de capacitación.

- La cantidad del personal que va a asistir a la capacitación.

- Los turnos del personal a capacitar.

- Verificar que se tengan todas las herramientas necesarias para la capacitación, es decir, el material, los accesorios y las instalaciones adecuadas.

- La persona encargada de ofrecer la capacitación.

- El método de difusión de la capacitación, es decir, el lugar, la fecha, la hora en caso de ser presencial.

- Crear guías rápidas de los equipos.

- Se debe de elaborar una estrategia con diversas herramientas para que el personal sea capacitado en su mayoría.

- Ejecutar el plan de capacitación y verificar por medio de evaluaciones el conocimiento adquirido en la capacitación.

- Documentar los resultados y el personal que asistió a la capacitación.

La Fig. 1 ilustra los pasos a seguir para elaborar el modelo de capacitación. 


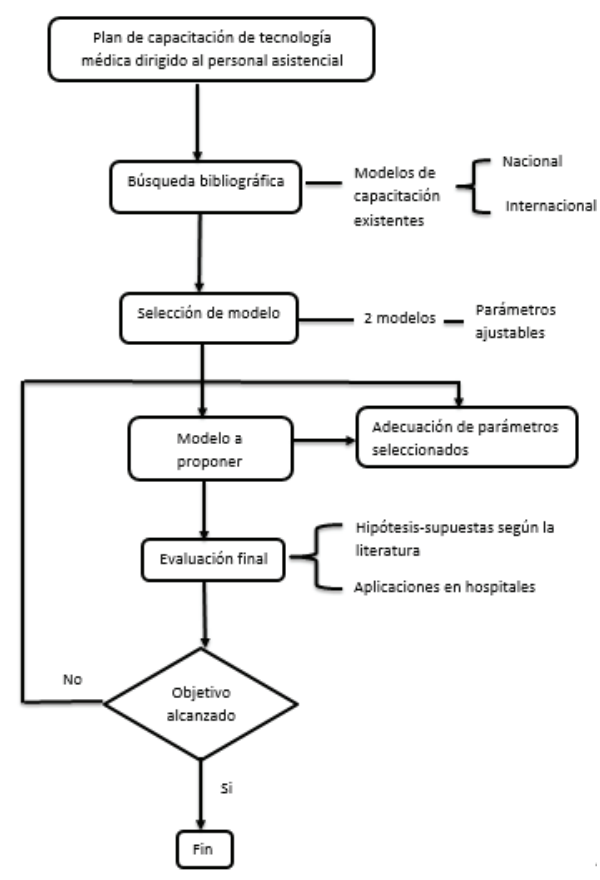

Fig 1. Diagrama de flujo de los pasos a seguir para elaborar el plan de capacitación del personal.

\section{RESULTADOS}

\section{A. Comparación de modelos.}

Para elaborar el modelo de capacitación destinado al personal asistencial se requiere realizar una comparación de los modelos actuales de capacitación a nivel nacional con referentes internacionales, para esto se utilizó como referente la metodología actual de capacitación de Newcastle Hospitals [4], entidad acreditada por la JCI, ente que identifica, mide y comparte con el mundo las mejores prácticas relacionadas con la calidad y la seguridad de los pacientes [5]. Y a su vez, como referente nacional se tomó una clínica de tercer nivel, del área metropolitana de Medellín, de la cual se logró obtener la información requerida para el estudio.

De acuerdo con los datos y la información recopilada de ambas instituciones prestadoras de servicios en salud, se realizó la toma de las cualidades más relevantes de cada una. De esta manera, en la Tabla 1, se visualiza los datos de Newcastle Hospitals, y en la Tabla 2, los datos de la clínica de tercer nivel.

Tabla 1. Información de Newcastle Hospitals.

\begin{tabular}{cc}
\hline Parámetros & The Newcastle Hospitals (NHS) \\
\hline Actualización de inventario & Entrada y salida de dispositivos \\
Revisión de inventario & Anualmente \\
Identificación de necesidades & Jefes de áreas
\end{tabular}

\begin{tabular}{cc} 
Detección mal uso del equipo & El equipo no se puede utilizar \\
Solicitud de capacitación & Jefes de áreas(personal asistencial) \\
Herramienta de solicitud & Escrito y por plataforma en línea \\
No se validan competencias & Se impide el uso del equipo \\
Ingreso de nuevo equipo & Se realiza una capacitación \\
No asistencia a la capacitación & Ocasiona acciones disciplinarias \\
Plataforma para capacitación & E-learning \\
Nivel de capacitación & Nivel de riesgo del equipo \\
Periodo sin hacer uso del & Se evalúan las competencias \\
Frecuencia de capacitación & Depende del nivel de riesgo \\
Tiempo de verificación inicial & 3 semanas \\
Evaluación de competencias & Presencial \\
Obligación de capacitación & Políticas de la entidad \\
Reporte de eventos adversos & Tecnovigilancia \\
\hline
\end{tabular}

Tabla 2. Información de la clínica de tercer nivel.

\begin{tabular}{cc}
\hline Parámetros & Clínica Tercer Nivel \\
\hline Actualización de inventario & $\begin{array}{c}\text { Entrada y salida de dispositivos } \\
\text { Anualmente }\end{array}$ \\
Identificación de necesidades & $\begin{array}{c}\text { Auxiliar del ingeniero jefe y } \\
\text { tecnólogos }\end{array}$ \\
Detección mal uso del equipo & Se programa la capacitación \\
aunque el personal hace uso de el \\
Solicitud de capacitación & Personal asistencial \\
Herramienta de solicitud & Teléfono y por plataforma en \\
línea
\end{tabular}

\section{B. Modelo}

El modelo inicialmente consiste en detectar las necesidades de capacitación, en primera instancia cuando se adquiere nueva tecnología [6], el área encargada de la adquisición de los nuevos dispositivos médicos debe de asegurar que el proveedor o fabricante de estos dispositivos brinde la capacitación por parte del personal capacitado y certificado en la tecnología, preferiblemente 
con una formación en pedagogía asegurando una adecuada capacitación inicial.

Otra situación es cuando la entidad posee la tecnología, en este punto se debe tener en cuenta indicadores de eventos adversos, el nivel de riesgo del equipo, solicitudes por parte de los jefes de cada área y los reportes de falla o duda recurrente del equipo.

Por último, el nuevo personal debe de ser evaluado en el tiempo de inducción, con el fin de tener una visión de aquellas falencias que posea, sabiendo de esta manera el enfoque de las capacitaciones.

Luego de elaborar el plan de capacitación se debe programar con los jefes de los departamentos con que regularidad se realizará la capacitación teniendo en cuenta las necesidades del personal y el área. Además, en que días y en que horario se llevarán a cabo las capacitaciones, esta programación debe contener la fecha en la que se realizará la capacitación, la hora, la duración, el lugar, el nombre del equipo, el modelo y la marca.

Y finalmente, se debe ejecutar el plan de capacitación donde se debe pasar la lista de asistencia al iniciar y finalizar la capacitación, aplicar un examen antes de iniciar y uno al finalizar la capacitación para medir los conocimientos adquiridos al personal capacitado, teniendo en cuenta que la evaluación final se aprueba por encima del $80 \%$, en caso de no aprobarse, el personal deberá repetir la evaluación dos días después con ayuda de la plataforma e-learning. El personal que no certifique que posee las cualidades necesarias para el uso seguro del equipo, no podrá hacer uso de él.

El paso a seguir posterior a la capacitación y su respectiva evaluación, es el análisis de los resultados para detectar las falencias y mejoras necesarias del plan actual de capacitación, ya que este plan se realizará de manera continua y permanente con el personal asistencial, por lo tanto todo el proceso deberá ser documentado y registrado. El modelo de capacitación se encuentra resumido en la Fig. 2 donde se muestran los pasos a seguir y los aspectos más importantes.

Para conceptualizar el modelo, se realizó una prueba teórica teniendo en cuenta datos de una unidad de cuidados intensivos. Esta área se simula con 674 equipos en total, distribuidos según su nivel de riesgo mediante la clasificación INVIMA (I, IIA, IIB y III), siendo nivel I 124 equipos distribuidos en 17 tecnologías diferentes, para el nivel IIA 371 equipos en 29 tecnologías, el nivel IIB cuenta con 104 equipos que representan 19 tecnologías y del nivel de más riesgo (nivel III) hay un total de 76 equipos en 7 diferentes tecnologías.

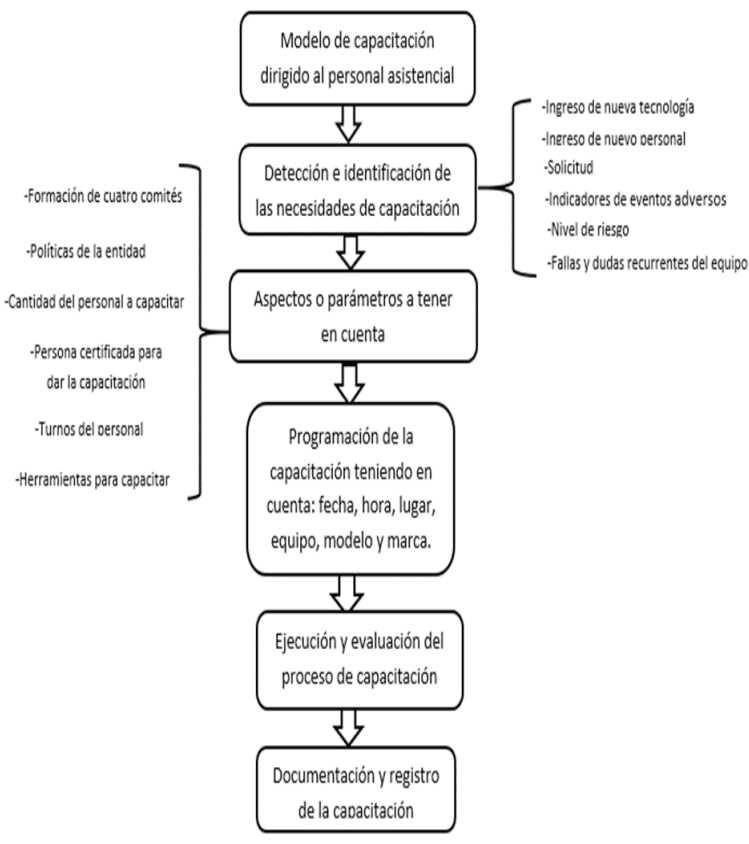

Fig. 2. Modelo de capacitación al personal asistencial.

La prueba consistió en comparar el total de horas de capacitación de un modelo donde el tiempo de capacitación es independiente del nivel de riesgo del equipo, es decir, para todas las tecnologías la capacitación tiene una duración de 90 minutos, respecto al modelo, en el cual el tiempo de capacitación se distribuye como se muestra en la Tabla 3. Estos datos se obtienen a partir de la revisión del modelo de capacitación del hospital local de nivel 3 que fue sujeto de prueba en la investigación.

Tabla 3. Distribución del nivel de riesgo.

\begin{tabular}{cc}
\hline Nivel de Riesgo & Tiempo de Duración [min] \\
\hline I & 30 \\
IIA & 45 \\
IIB & 90 \\
III & 150 \\
\hline
\end{tabular}

De acuerdo con ambos modelos, se calculó el total de horas requeridas para la capacitación de las tecnologías presentes en el área de acuerdo al tiempo asignado a cada tipo de capacitación, como se observa en la Tabla 4.

Con base a la distribución en horas de ambos modelos, se calculó el tiempo requerido por el personal asistencial dependiendo del cargo que tenga en la entidad prestadora de servicios en salud. La distribución anterior se muestra en la Tabla 5. 
Tabla 4 Distribución del nivel de riesgo.

\begin{tabular}{ccc|cc}
\hline \multicolumn{5}{c}{ Unidad de Cuidado Intensivo } \\
\hline Nivel de riesgo & Número & Porcentaje & Sin Riesgo & Con Riesgo \\
\hline I & 17 & $24 \%$ & 25,5 & 8,5 \\
IIA & 29 & $40 \%$ & 43,5 & 21,75 \\
IIB & 19 & $26 \%$ & 28,5 & 28,5 \\
III & 7 & $10 \%$ & 10,5 & 17,5 \\
Total: & 72 & $100 \%$ & 108 & 76,25 \\
\hline
\end{tabular}

Tabla 5. Horas de Capacitación para el personal.

\begin{tabular}{ccccc}
\hline Personal & Número & Por capacitación & $\begin{array}{c}\text { Horas sin } \\
\text { riesgo }\end{array}$ & $\begin{array}{c}\text { Horas con } \\
\text { riesgo }\end{array}$ \\
\hline Especialista & 3 & 1 & 117 & 138 \\
Médico & 4 & 2 & 144 & 100,5 \\
Enfermera & 8 & 2 & 276 & 121 \\
Auxiliar & 20 & 4 & 127,5 & 42,5 \\
Total & 35 & 9 & 664,5 & 402 \\
\hline
\end{tabular}

Finalmente, se realizó el cálculo del costo de la capacitación para el personal asistencial, para esto se hizo supuestos del valor de nómina de cada uno, el resultado se observa en la Tabla 6.

Tabla 6. Impacto del modelo en el costo de capacitación del personal asistencial.

\begin{tabular}{ccccc}
\hline Personal & $\begin{array}{c}\text { Salario } \\
\text { mensual }\end{array}$ & $\begin{array}{c}\text { Valor } \\
\text { hora }\end{array}$ & $\begin{array}{c}\text { Costo sin } \\
\text { riesgo }\end{array}$ & $\begin{array}{c}\text { Costo con } \\
\text { riesgo }\end{array}$ \\
\hline Especialista & $\$ 11.000 .000$ & $\$ 21.825$ & $\$ 2.553 .571$ & $\$ 3.011 .904$ \\
Médico & $\$ 4.200 .000$ & $\$ 8.333$ & $\$ 1.200 .000$ & $\$ 837.500$ \\
Enfermera & $\$ 3.000 .000$ & $\$ 5.952$ & $\$ 1.642 .857$ & $\$ 720.238$ \\
Auxiliar & $\$ 2.000 .000$ & $\$ 3.968$ & $\$ 505.952$ & $\$ 168.650$ \\
Total & $\$ 20.200 .000$ & $\$ 40.079$ & $\$ 5.902 .380$ & $\$ 4.738 .293$ \\
\hline
\end{tabular}

La diferencia en horas de capacitación es aproximadamente de 262 horas entre el modelo que considera el riesgo como un factor importante a tener en cuenta y el modelo que no lo tiene, adicional a esto en términos del valor de la nómina equivalente por asistir a los entrenamientos la diferencia entre el modelo que considera el riesgo y el modelo que no lo considera es de $\$ 1.164 .087$ lo que implica importantes ahorros en tiempo y dinero a tener en cuenta al momento de implementar un modelo de capacitación.

\section{DISCUSIÓN}

Para la seguridad del paciente y el usuario, la capacitación adecuada es crítica tanto para el personal asistencial como el personal técnico. El personal técnico y el gerente del departamento de ingeniería clínica tienen el doble de responsabilidad, debido a que deben informar, capacitar, programar y concientizar acerca de la importancia de tener un plan de capacitación. La capacitación no es una actividad elaborada en una sola ocasión, sino que es un proceso que debe realizarse de forma continua [7]. A su vez, el personal debe tener presente que el aprendizaje es importante para mejorar la fiabilidad y el éxito en el futuro de la solución a problemas además de brindar al paciente seguridad en el momento de ingresar a la entidad prestadora de servicios de salud.

La necesidad de capacitar se va ampliando más, si se toma en cuenta que un elevado porcentaje de los accidentes y daños en los equipos médicos tienen su origen en errores cometidos por los operadores. Es decir, que una capacitación adecuada al personal asistencial reduce a la larga la incidencia a la falla de los equipos médicos [8]. Dichos errores son difíciles de cuantificar debido a la falta de indicadores que midan este efecto, un futuro trabajo puede abarcar este aspecto para tener indicadores claros al respecto.

Uno de los parámetros más críticos para programar y realizar la capacitación es la plataforma utilizada para tal fin. Por medio de esta plataforma se busca tener herramientas tales como videos, formatos, imágenes, manuales y evaluaciones acerca de los dispositivos médicos que se encuentran en el área de interés, de esta manera el personal se encuentra en constante interacción con los dispositivos médicos.

Para detectar la necesidad de capacitación se deben emplean diversas estrategias, dentro de las cuales están las solicitudes enviadas por el personal, y por medio de indicadores de eventos adversos Vs cantidad de incidentes en donde se verifica que el fallo o el evento fue debido a un mal uso del equipo. Además, se recomienda que sea el jefe de cada departamento el encargado de verificar las necesidades de capacitación mediante estrategias de concientización al personal.

Otro de los aspectos críticos para realizar la capacitación, es la disponibilidad horaria del personal asistencial, debido a que este personal tiene una larga jornada laboral, además es difícil tener a todo el personal reunido para realizar la capacitación por el personal capacitado. En el caso del hospital de Newcastle la institución tiene una política en donde el personal debe asistir obligatoriamente a la capacitación programada. Una estrategia para la programación de la capacitación, en lo que respecta al tiempo del personal es elaborar dos capacitaciones en la jornada, para que de esta forma todo el personal pueda atender a la capacitación.

Por otro lado, es difícil controlar que el personal asistencial realice reportes de los incidentes adversos o los daños que pueda tener el equipo, esto muchas veces se 
presenta por el temor de recibir una sanción disciplinaria. Por lo que es importante crear estrategias en donde se concientice acerca de la importancia de realizar estos reportes para capacitar cuando se observe una falla o duda recurrente del personal que maneja el equipo [9].

Para finalizar, es importante, como se evidencia en la simulación realizada, la inclusión del riesgo de la tecnología como criterio importante cuando se ejecuta un plan de capacitación especificado en la Tabla 6, puesto que las diferencias entre un modelo que tenga en cuenta dicho criterio y uno que no son importantes en términos de tiempo y costo.

\section{Conclusiones}

En las diferentes instituciones prestadoras de servicios en salud, es necesario fortalecer los planes de capacitación, algunos factores que influyen en la necesidad de dicha estructuración son; la constante rotación de personal, el ingreso de nuevas tecnologías o la mala formación del personal asistencial en el uso de los dispositivos médicos que tienen a disposición para realizar su labor. Es por esto que se debe garantizar que el personal esté en todas las cualidades y cumpla con todos los requisitos para brindar una atención con el mínimo riesgo hacia el paciente, siendo primordial la concientización de cada uno de los integrantes de la planta del personal acerca del riesgo que implica no tener conocimiento del uso de los diferentes dispositivos médicos. [10]

Las instituciones prestadoras de servicios en salud, deben tener una política relacionada con el obligatorio cumplimiento las diferentes capacitaciones relacionadas con el uso de los dispositivos médicos, con lo cual la entidad garantiza que el personal que está manipulando los diversos dispositivos de las diferentes áreas poseen la formación necesaria en el uso de estos equipos. [11]

El plan de capacitación de cada entidad, sea pública o privada, con mucha o poca rotación del personal, debe de garantizar que el plan se realice de manera continua, atendiendo a las necesidades del personal asistencial, donde tendrán a disposición las herramientas necesarias para su educación en el manejo de aquellos dispositivos que se encuentran en el área de trabajo. Las herramientas van desde manuales, videos, conferencias, asesoría por parte de los ingenieros hasta una plataforma donde se puede tener acceso a toda la información acerca de los dispositivos, además, esta plataforma sirve para un control y evaluación de las competencias del personal en el uso de cada uno de los equipos requeridos en las áreas respectivas de trabajo.

La entidad debe de tener presente el nivel de riesgo de cada equipo en la programación de la capacitación de cada dispositivo médico, donde cada tecnología debe de ser tratada de manera independiente, respondiendo a las necesidades tanto del personal como del propio dispositivo para su adecuado uso, por lo tanto, el tiempo necesario de capacitación y el periodo con que se realizará las capacitaciones en el año, no serán programados de manera general, sino, que la programación deberá realizarse por grupos de riesgo (equipos que involucren el mismo nivel de riesgo), donde se tenga en cuenta las necesidades propias de estos dispositivos, lo que conlleva a la reducción de las horas necesarias para la capacitación, debido a que el personal recibirá la capacitación únicamente de aquellas tecnologías de interés.

\section{AgradeCIMIENTO}

Los autores agradecen la información ofrecida por la Clínica León XIII (IPS Universitaria)-Sede Medellín.

\section{REFERENCIAS}

[1]. Donalson L., Sukhmeet S., Ara D. Patient-Safety-Related Hospital Deaths in England: Thematic Analysis of Incidents Reported to a National Database, 2010-2012. Revista Pmed. 101371, 2014.

[2]. Gluck P. Seguridad del Paciente. Cierto Avance y Numerosos Retos. Revista Obstetricians and Gynecologists. 120, 1149-59, 2012.

[3]. Medicines \& Healthcare Products Regulatory Agency. Managing Medical Devices. Guidance for healthcare and social services organizations, Abril 2015.

[4]. Royal Cornwall Hospitals NHS. Medical Devices Training Policy. Volumen 1.9, Mayo 2013.

[5]. Joint Commission International. Estándares para la acreditación de Hospitales de la Joint Commission International. Consultado el 4 de junio de 2015 en: https: //www.jcrinc.com/assets/1/14/ EBJCI H14S_Sample_Pages.pdf. Ed 4, 2011.

[6]. Izquierdo E. Manual de capacitación de personal de mantenimiento hospitalario. Fecha de consulta 5 de Junio de 2015. Ministerio de salud pública república de Guatemala.

[7]. Word Health Organization. Medical equipment maintenance programme overview: WHO Medical device technical series. Junio 2011.

[8]. López M., Ayala R. Guía de Procedimientos para un Centro Estatal de Ingeniería Biomédica (CEDIB). Centro Nacional de Excelencia Tecnológica en Salud. 1 Ed. México, 2013.

[9]. Ministerio de salud pública. Guía para el reporte de eventos adversos por el fabricante. Consultado el 4 de Junio de 2015 en: www.eqmed.sld.cu/Documents/Guias/gt16.pdf.

[10]. Elsarnagawy T., Alhamwi A. Quality assurance in biomedical engineering COOP-educational training program: Planning, implementation and analysis. Journal of King Saud University Engineering Sciences, 23(2), 119-122, 2011.

[11]. Kontodimopoulos N., Pallikarakis N., Christov I., Daskalov I. In-house development of test equipment for quality control and training. Case study: a prototype ECG simulator-tester. Medical Engineering \& Physics, 20(10), 717-721, 1999. 